\title{
Protéger les enfants canadiens des conséquences de la quatrième vague de la pandémie de COVID-19
}

\author{
Stephen B. Freedman MDCM MSc, James D. Kellner MD MSc
}

Citation : CMAJ 2021 September 27;193:E1500-2. doi : 10.1503/cmaj.211513-f

Voir la version anglaise de l'article ici : www.cmaj.ca/lookup/doi/10.1503/cmaj.211513; voir l'article connexe en anglais ici : www.cmaj. ca/lookup/doi/10.1503/cmaj.210053; et en français ici : www.cmaj.ca/lookup/doi/10.1503/cmaj.210053-f

A lors que la quatrième vague de la pandémie de COVID19 balaie le Canada, il est de la plus haute importance que nous comprenions les conséquences de l'infection par le SRAS-CoV-2 chez les enfants et que nous intervenions adéquatement. Même si des vaccins très efficaces contre l'infection sont actuellement disponibles pour les enfants de 12 ans et plus, aucun n'a encore été approuvé pour les plus jeunes; leur date d'approbation pour cette population reste incertaine, et leur taux d'adoption, imprévisible. Dans ce contexte, une étude connexe réalisée à partir des données ${ }^{1} \mathrm{du}$ Programme canadien de surveillance pédiatrique (PCSP) présente des renseignements sur la fréquence des hospitalisations et les caractéristiques des enfants atteints de la COVID-19 qui sont hospitalisés, des indicateurs clés du fardeau de la maladie chez les enfants. Toutefois, les politiques publiques doivent tenir compte d'autres considérations, notamment les répercussions de l'arrivée de nouveaux variants du SRAS-CoV-2 (p. ex., variant Delta), l'incidence du syndrome inflammatoire multisystémique chez les enfants (SIMS-E) et des manifestations post-COVID, ainsi que la disponibilité et l'adoption des vaccins contre la COVID-19.

L'étude connexe ${ }^{1}$ démontre clairement que les enfants ont été relativement épargnés durant les 2 premières vagues de la pandémie. Drouin et ses collègues notent qu'au 31 décembre 2020, on avait signalé 308 hospitalisations d'enfants infectés par le SRAS-CoV-2 à l'Agence de la santé publique du Canada. Par l'entremise du système de signalement du PCSP, les auteurs ont analysé les dossiers de 264 enfants hospitalisés en raison d'une infection par le SRAS-CoV-2 aiguë et pour lesquels des données détaillées avaient été soumises. Parmi ces cas, on indiquait que seulement 150 (57\%) avaient été hospitalisés en raison de la COVID-19. Ce faible nombre témoigne de la nature bénigne de l'infection par le SRAS-CoV-2 chez les enfants et de l'efficacité des mesures sanitaires mises en place pour prévenir la propagation à grande échelle de la maladie chez les enfants.

\section{Points clés}

- Peu d'enfants ont été hospitalisés au Canada en raison de la COVID19 au cours des premières vagues de la pandémie; toutefois, les enfants hospitalisés étaient souvent gravement malades.

- Les conséquences à plus long terme de l'infection par le SRASCoV-2 (p. ex., syndrome inflammatoire multisystémique et manifestations post-COVID) peuvent avoir des répercussions plus graves sur la santé des enfants que la maladie aiguë, et le fardeau de ces affections doit être considéré et étudié, particulièrement dans le contexte du variant Delta.

- Étant donné que les enfants canadiens de moins de 12 ans ne peuvent pas encore recevoir de vaccin contre la COVID-19, il est important de s'assurer que tous ceux qui y sont admissibles se font vacciner et de maintenir les autres mesures d'atténuation, comme le port du couvre-visage et l'amélioration de la ventilation afin de protéger les enfants à l'aube d'une nouvelle année scolaire.

Une analyse des données tirées des dossiers médicaux électroniques effectuée en septembre 2020 qui incluait plus de 135000 personnes de moins de 25 ans aux États-Unis a révélé que $7 \%$ des personnes infectées par le SRAS-CoV-2 avaient été hospitalisées, dont $28 \%$ dans une unité de soins intensifs (USI) ${ }^{2}$. Même si l'étude connexe ${ }^{1}$ ne précise pas la proportion d'enfants ayant contracté le SRASCoV-2 qui ont dû être hospitalisés, les auteurs mentionnent que parmi les enfants hospitalisés pour la COVID-19, 21\% ont dû être traités dans une USI et $6 \%$ ont eu besoin de ventilation mécanique. Selon les plus récentes données canadiennes (en date du 29 août 2021), la proportion totale d'enfants ayant contracté le SRAS-CoV-2 qui ont été hospitalisés est faible (0,5\%); toutefois, $11 \%$ d'entre eux ont dû être admis dans une $\mathrm{USI}^{3}$. Cette proportion relative d'hospitalisations pédiatriques pour une maladie grave est inquiétante. Comparativement à d'autres maladies respiratoires pédiatriques, le taux d'hospitalisation en USI excède ceux que l'on observe chez les enfants atteints de bronchiolite $^{4}$ et de grippe $\mathrm{H} 1 \mathrm{~N} 1$ saisonnière et pandémique ${ }^{5}$. 
Par ailleurs, les cas signalés dans l'étude connexe ${ }^{1}$ sont survenus avant l'émergence du variant Delta du SRAS-CoV-2, ce qui nous oblige à réévaluer le risque. Le variant Delta est plus facilement transmissible que les souches précédentes, et les données émergentes décrivent des taux de morbidité plus élevés à tous les âges, y compris chez les enfants. Une étude de cohorte qui incluait tous les patients atteints de COVID-19 en Angleterre au printemps 2021 (dont 30\% étaient âgés de moins de 20 ans) a révélé que le risque d'hospitalisation était plus de 2 fois plus élevé chez les sujets infectés par le variant Delta que chez les sujets infectés par le variant Alpha, particulièrement s'ils n'avaient pas été vaccinés ${ }^{6}$. Cela soulève des inquiétudes au sujet de l'impact du variant Delta sur les enfants au cours de l'automne et de l'hiver 2021-2022, qu'il se présente seul ou en concomitance avec d'autres maladies respiratoires.

L'infection par le SRAS-CoV-2 peut aussi donner lieu au SIMS-E, une complication post-infectieuse. L'incidence du SIMS-E est estimée à 316/1000 000 de cas de SRAS-CoV-2 (1/3165 enfants ayant contracté le SRAS-CoV-2) ${ }^{7}$. Les enfants qui présentent un SIMS-E sont en général très malades, près de $75 \%$ ayant nécessité un séjour en USI et $2 \%$ en étant décédés ${ }^{8}$. On ignore encore si la vague actuelle, causée par le variant Delta, combinée à des taux insuffisants de vaccination dans la population générale, à l'absence de vaccination chez les moins de 12 ans et à l'assouplissement des mesures sanitaires entraînera une augmentation des cas de SIMS-E. Cela dit, si on se fie aux vagues précédentes, le SIMS-E atteint un pic dans les 4 à 6 semaines qui suivent une poussée soudaine du nombre cas d'infections au SRAS-CoV-2; on peut donc s'attendre à une augmentation similaire chez les enfants durant la quatrième vague de la pandémie au Canada.

Il faut également tenir compte de l'éventail des manifestations post-COVID. Même si les effets à long terme ont été bien caractérisés dans les cas graves de COVID-19 aiguë chez les adultes, ils sont de plus en plus souvent signalés dans des cas initialement bénins d'infection par le SRAS-CoV-2. La prévalence des manifestations post-COVID semble plus faible chez les enfants que chez les adultes, bien qu'on ne dispose pas d'études définitives à ce sujet, mais on note une tendance résolument à la hausse de la proportion d'enfants ayant contracté le SRAS-CoV-2 qui font état de symptômes persistants plusieurs mois après l'infection ${ }^{9}$, par rapport aux témoins (enfants ayant obtenu des résultats négatifs aux tests de dépistage $)^{9,10}$. Les preuves que des manifestations postCOVID sont aussi présentes chez les enfants s'accumulent : il faudra donc en tenir compte au moment de quantifier le fardeau de l'infection par le SRAS-CoV-2 chez les enfants.

Ensemble, ces enjeux rappellent l'importance de maintenir les efforts de prévention de l'infection par le SRAS-CoV-2 chez les enfants. Le Canada se trouve à un tournant de la pandémie de COVID-19 : la quatrième vague gagne rapidement du terrain alors qu'une nouvelle année scolaire débute. La plupart des enfants fréquenteront l'école en personne, et comme les enfants de moins de 12 ans ne sont pas admissibles à la vaccination, ils constituent un groupe particulièrement vulnérable. Il est impératif d'éviter les fermetures d'école pour réduire les répercussions sur la santé mentale et la réussite scolaire des enfants. À l'heure actuelle, toutefois, on note que les mesures préventives varient considérablement à l'intérieur des écoles et autres milieux fréquentés par les enfants partout au Canada. La propagation du SRAS-CoV-2 par voie aérienne est particulièrement problématique en contexte scolaire, en raison de la ventilation inadéquate de certains sites : une personne infectée pourrait y être présente pendant de longues périodes, ce qui peut mener à l'accumulation d'une quantité suffisante de particules aériennes pour permettre la transmission de l'infection. Il est vrai qu'au cours des premiers mois de la pandémie, on manquait de données robustes appuyant le port du couvrevisage, mais on sait à présent que dans la communauté, il réduit efficacement la propagation du SRAS-CoV-2 et, dans une moindre mesure, protège la personne qui le porte ${ }^{11}$. Malgré l'inconfort associé au port du masque, ce dernier n'influe pas substantiellement sur la capacité des enfants de reconnaître les émotions et d'y répondre ${ }^{4}$, et constitue une importante mesure sanitaire qu'on devrait appliquer pour protéger les enfants.

Même si, comme l'ont montré les auteurs de l'étude connexe ${ }^{1}$, les conséquences de la COVID-19 aiguë chez les enfants ont été limitées lors des premières phases de la pandémie, on doit tenir compte des répercussions directes et indirectes de l'infection par le SRAS-CoV-2 chez les enfants au moment d'établir des politiques de santé publique. Ces délibérations doivent tenir compte des répercussions à court et à long terme des politiques publiques sur le bien-être physique, mental et social des enfants. Même si l'on voit la lumière au bout du tunnel, il faut continuer de protéger les enfants du Canada, puisqu'ils risquent d'être les derniers à atteindre le fil d'arrivée.

\section{Références}

1. Drouin O, Moore Hepburn C, Farrar DS, et al. Characteristics of children admitted to hospital with acute SARS-CoV-2 infection in Canada in 2020. CMAJ 2021;193:E1483-93.

2. Bailey LC, Razzaghi H, Burrows EK, et al. Assessment of 135794 pediatric patients tested for severe acute respiratory syndrome coronavirus 2 across the United States. JAMA Pediatr 2021;175:176-84.

3. COVID-19 daily epidemiology update. Ottawa: Government of Canada; 2021. Accessible ici : https://health-infobase.canada.ca/covid-19/epidemiological -summary-covid-19-cases.html (consulté le 29 août 2021).

4. Oakley $\mathrm{E}$, Chong V, Borland $\mathrm{M}$, et al. Intensive care unit admissions and ventilation support in infants with bronchiolitis. Emerg Med Australas 2017;29:421-8.

5. Tran D, Vaudry W, Moore DL, et al. Comparison of children hospitalized with seasonal versus pandemic influenza A, 2004-2009. Pediatrics 2012;130:397-406.

6. Twohig KA, Nyberg T, Zaidi A, et al. Hospital admission and emergency care attendance risk for SARS-CoV-2 delta (B.1.617.2) compared with alpha (B.1.1.7) variants of concern: a cohort study. Lancet Infect Dis 2021;S1473-3099:00475-8.

7. Payne AB, Gilani Z, Godfred-Cato S, et al. Incidence of multisystem inflammatory syndrome in children among US persons infected with SARS-CoV-2. JAMA Netw Open 2021;4:e2116420.

8. Feldstein LR, Tenforde MW, Friedman KG, et al. Characteristics and outcomes of US children and adolescents with multisystem inflammatory syndrome in children (MIS-C) compared with severe acute COVID-19. JAMA 2021;325: 1074-87.

9. Molteni E, Sudre $\mathrm{CH}$, Canas LS, et al. Illness duration and symptom profile in symptomatic UK school-aged children tested for SARS-CoV-2. Lancet Child Adolesc Health 2021 Aug. 3 [cyberpublication avant impression]. doi: https://doi. org/10.1016/S2352-4642(21)00198-X

10. Stephenson T, Pinto Pereira S, Shafran R, et al.; CLoCK Consortium. Long COVID the physical and mental health of children and non-hospitalised young people 3 months after SARS-CoV-2 infection; a national matched cohort study (The CLoCk study). In Review [préimpression] 2021 Aug. 10. doi: 10.21203/rs.3.rs-798316/v1.

11. Johansson MA, Quandelacy TM, Kada S, et al. SARS-CoV-2 transmission from people without COVID-19 symptoms. JAMA Netw Open 2021;4:e2035057-e.

12. Ruba AL, Pollak SD. Children's emotion inferences from masked faces: implications for social interactions during COVID-19. PLoS One 2020;15:e0243708. 
Intérêts concurrents : Les travaux de Stephen Freedman sont financés par la chaire de recherche en santé et bien-être des enfants de la Fondation de l'Hôpital pour enfants de l'Alberta. James Kellner est membre du comité de surveillance de la sécurisation des données pour un essai clinique sur un vaccin contre la COVID-19 commandité par la Vaccine and Infectious Disease Organization de l'Université de la Saskatchewan; membre de l'Équipe de direction, coprésident du Groupe de travail sur les études sur le terrain et président du Réseau pédiatrique pour le Groupe de travail sur l'immunité face à la COVID-19; conseiller auprès du sous-ministre de la Santé et de la médecin hygiéniste en chef, en tant que membre du Comité consultatif de l'Alberta sur l'immunisation. Aucun autre intérêt concurrent n'a été déclaré.

Cet article a été sollicité et il n'a pas été révisé par des pairs.

Affiliations : Institut de recherche de l'Hôpital pour enfants de l'Alberta, Faculté de médecine Cumming, Université de Calgary, Calgary, Alb.

Collaborateurs : Stephen Freedman a rédigé l'ébauche du travail. James Kellner en a révisé de façon critique le contenu intellectuel important. Les deux auteurs ont contribué à la conception et à la modélisation du travail, ont donné leur approbation finale pour la version destinée à être publiée et assument l'entière responsabilité de tous les aspects du travail.

Propriété intellectuelle du contenu : Il s'agit d'un article en libre accès distribué conformément aux modalités de la licence Creative Commons
Attribution (CC BY-NC-ND 4.0), qui permet l'utilisation, la diffusion et la reproduction dans tout médium à la condition que la publication originale soit adéquatement citée, que l'utilisation se fasse à des fins non commerciales (c.-à-d., recherche ou éducation) et qu'aucune modification ni adaptation n'y soit apportée. Voir : https://creativecommons.org/licenses/by-nc -nd/4.0/deed.fr.

Financement : Stephen Freedman a reçu des subventions des agences suivantes pour réaliser des travaux sur le SRAS-CoV-2 : Santé Canada (Accord sur la relance sécuritaire), Instituts de recherche en santé du Canada (IRSC; Nouveaux besoins prioritaires en recherche sur la COVID-19; Subvention de fonctionnement : Financement supplémentaire - nouveau variant du SRAS-CoV-2 : Intervention de recherche rapide contre la COVID19 (volet 1); Intervention de recherche rapide contre la COVID-19Réponses en matière de politique sociale et de santé publique; COVID-19Gestion clinique), Agence de la santé publique du Canada (Fonds de partenariat d'immunisation - Programme de financement pour les enjeux émergents) et Services de santé Alberta - Université de Calgary - Fonds de recherche clinique. James Kellner a reçu des subventions des IRSC, du Canadian Immunization Research Network, de la BC Lung Association, de Genome Alberta et de la Fondation de l'Hôpital pour enfants de l'Alberta, ainsi que de Pfizer, GlaxoSmithKline, Merck et Moderna.

Correspondance : Stephen Freedman,

Stephen.Freedman@albertahealthservices.ca 